\title{
Controlling competition between crystallization and glass formation in binary colloids with an external field
}

\author{
C Patrick Royall ${ }^{1,2}$, Esther C M Vermolen ${ }^{3}$, Alfons van Blaaderen ${ }^{3}$ \\ and Hajime Tanaka ${ }^{2}$ \\ ${ }^{1}$ School of Chemistry, University of Bristol, Bristol BS8 1TS, UK \\ ${ }^{2}$ Institute of Industrial Science, University of Tokyo, 4-6-1 Komaba, Meguro-ku, \\ Tokyo 153-8505, Japan \\ ${ }^{3}$ Debye Institute for NanoMaterials Science, Utrecht University, Princetonplein 5, \\ 3584CC, Utrecht, The Netherlands \\ E-mail: paddy.royall@bristol.ac.uk and tanaka@iis.u-tokyo.ac.jp
}

Received 25 April 2008, in final form 6 June 2008

Published 10 September 2008

Online at stacks.iop.org/JPhysCM/20/404225

\begin{abstract}
The mechanism by which a liquid may become arrested, forming a glass, is a long-standing problem. So far no clear structural mechanism has been found. One promising approach is to use real space analysis of colloidal dispersions at the single-particle level to reveal local structural details which are inaccessible to many experimental techniques. Here we report a simple method to control glass formation which enables us to tackle the competition between crystallization and vitrification. While monodisperse colloidal hard spheres may readily crystallize, polydisperse suspensions tend to form glassy structures. We exploit the difference in sedimentation velocities of colloidal particles of different sizes, leading to a sediment which changes continuously in composition as a function of height, revealing glassy and crystalline states.
\end{abstract}

(Some figures in this article are in colour only in the electronic version)

\section{Introduction}

When a liquid is cooled sufficiently, and is unable to access the thermodynamic equilibrium state, it may undergo dynamical arrest and become a glass [1]. Among the key unanswered questions in condensed matter is the physical mechanism underlying this glass transition. Although possible dynamical mechanisms have received considerable attention [2], a structural mechanism has so far been hard to identify [3-5]. A central issue concerning a structural mechanism is the concept of competition between crystallization and vitrification [6-9]. However, the identification of (local) signatures such as medium range crystalline order or energetically locally favoured structures that might demonstrate such a structural mechanism is very challenging for reciprocal space techniques such as x-ray scattering which usually average over many particles.

However, atoms and molecules are not the only systems which undergo dynamical arrest. Colloidal suspensions are recognized as mesoscopic 'model atoms' because they have a well-defined thermodynamic temperature, and also form glasses. Furthermore, under the appropriate conditions, colloidal suspensions can provide a remarkably good approximation to perhaps the simplest system known to exhibit a (structural) glass transition: hard spheres [10]. A significant breakthrough thus came when micron-sized colloidal hard spheres were resolved in $3 \mathrm{D}$, at the singleparticle level, using confocal microscopy [11], providing a level of detail usually available only to computer simulation. Along with a number of structural measurements, such as local fivefold symmetry [11, 12], long thought to occur in dynamically arrested states [13], it was also possibly to directly measure dynamic heterogeneity $[14,15]$.

Although experimental colloidal systems are inherently polydisperse, in computer simulations it has been shown that monodisperse hard spheres are very poor glass formers and instead crystallize [16, 17]. Indeed the shape of the distribution that determines the polydispersity has profound 
consequences for the crystallization rate [18]. In simulations of model atomic glass formers, crystallization may be avoided by the use of a binary system [19], although the situation can be more complex, as binary hard spheres, for example, may form a substitutional or binary crystal or, at higher densities, ultimately segregate and crystallize [20], evidence for which has also been found experimentally [21]. While the effects of polydispersity upon crystallization are subtle and not yet fully understood [18], it is clear that the use of binary systems can significantly inhibit crystallization, and may thus be a fruitful avenue for the exploration of the competition between crystallization and vitrification [7-9]. Furthermore, the correlation between local (dis)order and dynamics, recently accessed in computer simulations [22], may shed new light on the origins of dynamical arrest. Preliminary experimental work on a binary hard-sphere-like system with a size ratio 0.84 also showed a breakdown in long-ranged translational order [23].

Of central importance to the use of binary systems as a means to control the balance between crystallization and vitrification is the necessity to change the composition. The subject of this work is to introduce and demonstrate a conceptually simple and technically straightforward method to continuously vary the composition in a colloidal hard-sphere glass: gravity. We wish to underline that our main aim here is to demonstrate an experimental method by which samples may be conveniently prepared at a range of compositions. For this reason, we present mainly $2 \mathrm{D}$ results on a $3 \mathrm{D}$ system. However, we confirm the validity of our technique by comparing our 2D approach with a sample analysed in $3 \mathrm{D}$. We begin by discussing the experimental approach, techniques employed and present results which demonstrate the applicability of this method, concluding with a discussion of points for further investigation.

\section{Methods}

In a gravitational field, an isolated spherical colloid settles under gravity, at an average (Stokes') velocity

$$
u_{0}=\frac{m g}{3 \pi \eta \sigma}
$$

where $m=\left(4 \pi a^{3}\right) \delta \rho / 3$ is the buoyancy with $\delta \rho$ the mass density difference between the colloids and the solvent and $\sigma$ the diameter. $g$ is the acceleration due to gravity and $\eta$ is the viscosity of the solvent. This expression leads to a quadratic dependence of the sedimentation rate upon the particle size. Thus, in the case of a binary colloidal suspension, we may expect the sedimentation rate of large colloids to exceed that of the smaller, leading to a sediment rich in large particles at the bottom, with an increasing concentration of small particles as a function of height.

Although equation (1) is only valid in the dilute limit, and indeed rather dilute systems with more extreme size ratios are predicted to exhibit non-trivial equilibrium sedimentation profiles [24], in this case of a concentrated binary system of similarly sized colloids the system behaved as expected.

We exploit this size dependence of sedimentation rates to control the crystallization and vitrification in a suspension of
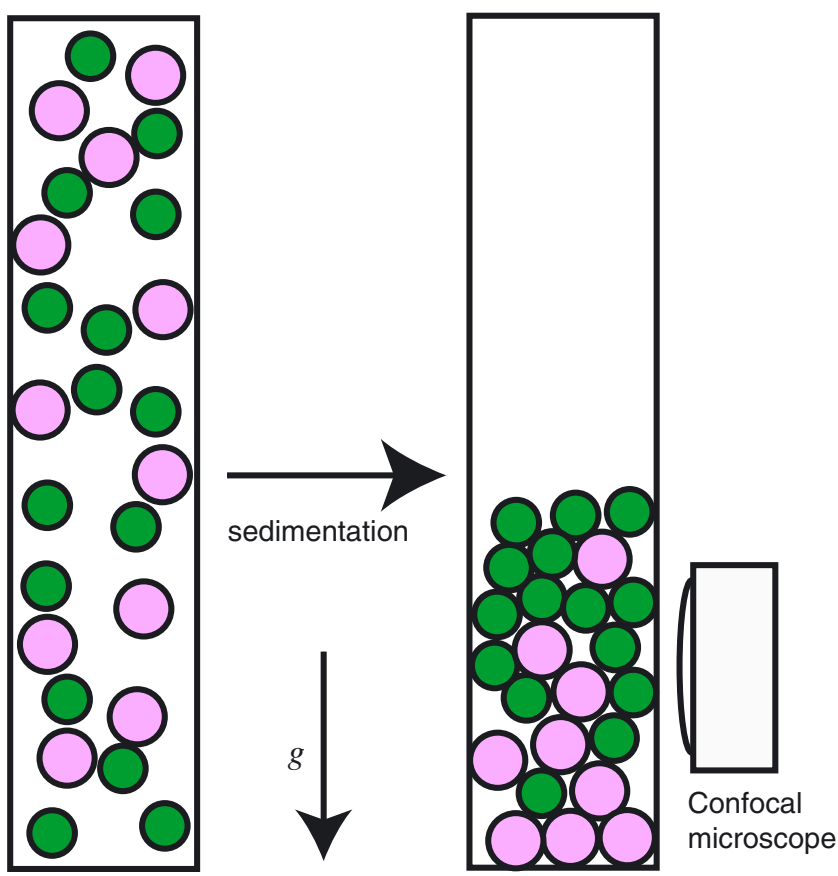

Figure 1. A schematic of the experimental set-up: colloids are allowed to sediment, and are subsequently imaged with a confocal microscope rotated through $90^{\circ}$. The increased sedimentation rate of the larger (light coloured) colloids leads to a continuously varying composition in the sedimentation profile. For simplicity the illustration is drawn for the case that the colloids are heavier than the solvent. In this work, the colloids are lighter than the solvent, and therefore cream rather than sediment.

nearly hard-sphere colloids. The sediment of a mixture of two colloid species is therefore expected to change continuously as a function of height. At the bottom, the larger particles are expected to dominate, while at intermediate heights, more symmetric mixtures are found, as shown schematically in figure 1. Finally, towards the top the sediment should be largely comprised of smaller particles. The polydispersity of each species is around 0.04, as determined with static light scattering, such that both species crystallize in isolation, but upon mixing crystallization may be inhibited.

\subsection{Experimental details}

We use a colloidal model system of polymethyl methacrylate, in a solvent mixture of cyclohexyl bromide (CHB) and cisdecalin [25], in which we set the density difference between the solvent mixture and the colloids such that the sedimentation rate in the concentrated suspension is around $1 \mathrm{~cm} \mathrm{day}^{-1}$ [26]. Hard-sphere-like interactions are obtained with the addition of tetrabutyl ammonium bromide salt [25]. We consider two systems. In the first, the large particles $\left(\sigma_{R}=2.16 \mu \mathrm{m}\right.$ diameter) are labelled with rhodamine isothicyanate (shown as red) and the smaller $\left(\sigma_{N}=2.0 \mu \mathrm{m}\right)$ with 7-nitrobenzo-2-oxa1,3-diazol (NBD), shown as green [27]. The size ratio of small to large particles $q$ is thus 0.92 . The initial volume fraction was 0.2 , comprised of $0.1 \mathrm{NBD}$ and 0.1 rhodamine particles. For this system we performed an analysis at different heights, exploring the effects of the compositional changes. 
In each set of experiments, the colloidal suspension was placed in a capillary of internal dimensions $0.1 \times 1 \mathrm{~mm}$ and left to settle for 14 days. The total height of the system was $2 \mathrm{~cm}$. The sediment was then imaged with a Leica TCS NT confocal microscope, operated at excitation wavelengths of $488 \mathrm{~nm}$ for the NBD and $568 \mathrm{~nm}$ for the rhodamine particles. The experimental set-up is sketched in figure 1 .

With the second system, we performed a $2 \mathrm{D}$ and $3 \mathrm{D}$ analysis. In this case, we used $\left(\sigma_{G}=2.0 \mu \mathrm{m}\right.$ diameter $)$ and $\left(\sigma_{R}=2.40 \mu \mathrm{m}\right.$ diameter), with comparable polydispersity to the above. The size ratio was thus $q=0.80$. In fact, in this system the smaller particles were labelled with rhodamine, while the larger were labelled with NBD. However, we depict larger particles in red and smaller particles in green throughout. Here the initial volume fraction was 0.23 and the internal dimensions of the capillary were $0.1 \mathrm{~mm} \times 2 \mathrm{~mm}$.

All images were taken within the sediment, and we detected no change in overall packing fraction. We return to the issue of overall volume fraction in the concluding section. All data presented here were taken from one sample, to underline the simplicity of this technique to scan a range of compositions quickly and easily. For the 2D analysis, images were taken as 2D optical slices, and analysed using methodology similar to that of Crocker and Grier [28] to yield coordinates for each particle. Although we imaged typically $20 \mu \mathrm{m}$ away from the glass wall, even at these distances, the wall produces some orientation of the crystal planes, (figure 4). In the case of the $3 \mathrm{D}$ analysis, we took a stack of 3D images some $155 \mathrm{~nm}$ apart and analysed these as previously described [11, 29].

\subsection{Analysis and order parameters}

Radial distribution function. The radial distribution function $g(r)$ was calculated as $g(r)=\frac{1}{2 \pi r \Delta r \rho(N-1)} \sum_{i \neq k} \delta\left(r-\left|\vec{r}_{i k}\right|\right)$, which is the ratio of the ensemble average of the number of particles in the region $r \sim r+\Delta r$ to the average number density $\rho=N / L^{2}$ where $N$ is the total number of particles, $L$ is the (square) image length and $\delta$ is the Dirac delta function.

Characterization of local bond-orientational order. To characterize the local structure, we calculate a complex sixfold bond-orientational order parameter for each particle [30]:

$$
\psi_{6}^{i}=\frac{1}{n_{i}} \sum_{m=1}^{n_{i}} \mathrm{e}^{\mathrm{j} 6 \theta_{m}^{i}}
$$

where $n_{i}$ is the number of nearest neighbours of particle $i$ and $\mathrm{j}=\sqrt{-1}$. $\theta_{m}^{i}$ is the angle between $\vec{r}_{m i}$ and the $x$ axis, where particle $m$ is a neighbour of particle $i$. We identify the neighbouring coordinates with a Voronoi construction. The value of $\psi_{6}^{i}$ is equal to 1 if all the particles sit on a hexagonal lattice. However, it approaches zero when the structure is disordered. The spatial correlation of $\psi_{6}^{i}$ is calculated as

$$
g_{6}(r)=\frac{L^{2}}{2 \pi r \Delta r N(N-1)} \sum_{i \neq k} \delta\left(r-\left|\vec{r}_{i k}\right|\right) \psi_{6}^{i} \psi_{6}^{k *} .
$$

The spatial correlation of the bond-orientational order can then be characterized by $g_{6}(r) / g(r)$.
Similarly, in the 3D case, we determine the bondorientational parameter $q_{6}$, for each particle, which is given by

$$
q_{6} \equiv \frac{1}{N_{b}(i)} \sum_{j=1}^{N_{b}(i)} Y_{6 m}\left(\mathbf{r}_{\mathbf{i j}}\right),
$$

where $Y_{6 m}$ are spherical harmonics [31].

The spatial correlation of $\psi_{6}^{i}$ is calculated as

$$
g_{6}^{3}(r)=\frac{L^{2}}{2 \pi r \Delta r N(N-1)} \sum_{i \neq k} \delta\left(r-\left|\vec{r}_{i k}\right|\right) q_{6}^{i} q_{6}^{k *} .
$$

As in the 2D case, the spatial correlation of the bondorientational order can then be characterized by $g_{6}^{3}(r) / g(r)$. However, we note that, unlike $\phi_{6}$, the value of $q_{6}$ is approximately 0.58 for face-centred cubic (FCC) and slightly lower for hexagonal close-packed HCP crystalline order [31].

\section{Results and discussion}

\subsection{Comparison of $2 D$ and $3 D$ data}

We begin our results and analysis by considering the differences between a 2D and 3D analysis. Now a 2D analysis of an amorphous phase cannot a priori discriminate between particles whose $z$ coordinate differs (figure 2). Although one may introduce schemes with a lower intensity threshold, which might be expected to provide a measure of the $z$ coordinate, these have yielded little success. Furthermore, one is forced to calibrate every new batch of colloidal particles (as the degree by which a particle loses intensity upon moving away from the focal plane relative to its diameter is a strong function of its size), for each set of imaging parameters. Even within one batch, there are some fluctuations in dye distribution between individual particles, not to mention the size polydispersity inherent in colloidal systems. Finally, there is no a priori way to know if the particle is above or below the plane. The consequences of this discussion are illustrated in figure 2. Such considerations apply somewhat less to colloidal crystals, since the crystal planes are typically formed parallel to the glass wall of the capillary. Thus we can track the coordinate of the particles in that crystal plane which is aligned with the image plane.

We argue, therefore, that a 2D analysis can yield information about the general structure, rather than providing a truly quantitative measurement of, for example, the radial distribution function $g(r)$ close to contact. However, for our current purposes, we seek to demonstrate a convenient experimental technique for generating a quasi-continuous change in composition. Since we expect that this change in composition will have the effect of moving the system from one state of long-ranged order (crystal of small particles) through a state without long-ranged order (the glassy mixture) to another state of long-ranged order (crystal of large particles), we simply require that we can confidently measure long-ranged order. Our discussion above concerning colloidal crystals suggests that long-ranged crystalline order may readily be detected in a $2 \mathrm{D}$ analysis. 


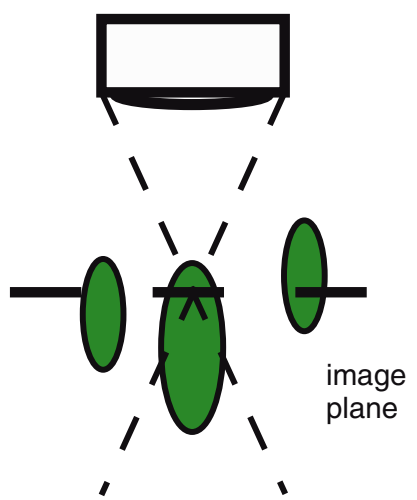

Figure 2. The perils of assigning a coordinate in the axial $(z)$ direction from a 2D image by considering the intensity in the imaging plane. All three particles may have a similar intensity in the $x y$ plane, yet their $z$ coordinates are rather different. The oval nature of the colloidal particles reflects the relatively poor resolution in the $z$ direction.

To compare the 2D and 3D analyses, we therefore consider the amorphous case. A mixed system is shown in figure 3(a), where the size ratio $q=0.80$. Here the volume fraction of large (red, $2.4 \mu \mathrm{m}$ particles) is around 0.20 , while that of the smaller particles (green, $2.0 \mu \mathrm{m}$ ) is around 0.44 . The system is clearly disordered, as is further evidenced by the correlation functions plotted in figure 3(b), following a 3D analysis. None shows any sign of long-ranged order. The $2 \mathrm{D}$ analysis shown in figure 3(c) shows similar qualitative behaviour. There is some difference in the local structure of the pair correlation functions between 3D (figure 3(b)) and 2D (c). This may be related to relatively larger tracking errors in the $3 \mathrm{D}$ case, due to the poorer resolution in the $z$ direction [29], or to the rather more limited level of information in the 2D case, as discussed above. Note, however, that both $3 \mathrm{D}$ and $2 \mathrm{D}$ data show a clear absence of long-ranged order.

\section{2. $2 D$ analysis of compositional changes}

Confocal micrographs are shown in figure 4 which reveal the overall structure and composition of the system, for which the size ratio $q=0.92$. The two species are readily distinguished using different fluorescent channels. When one species is in the majority (figures 4(a) and (f)), a substitutional crystal is found, consistent with simulation results [20]. More symmetric mixtures show a significant increase in disorder, in particular figure $4(\mathrm{c})(z=3.3 \mathrm{~mm})$ suggests a breakdown in long-ranged crystalline order [22].

Having demonstrated the basic principle of this method, we now turn our attention to a more quantitative analysis. We stress that the work presented here is intended to demonstrate the general principle, and therefore we are content to restrict ourselves to a 2D analysis, which, although it does not yield all the information, nevertheless provides some reliable pointers as to the usefulness of this technique, particularly whether or not we can control long-ranged order through compositional changes. We begin by considering the composition as a function of height, as shown in figure 5 . Here the area fraction $\phi_{i}$ is defined as $\phi_{i}=\left(\pi \sigma_{i}^{2} / 4\right) N_{i} / L^{2}$, where $\sigma_{i}$ is the diameter (a)

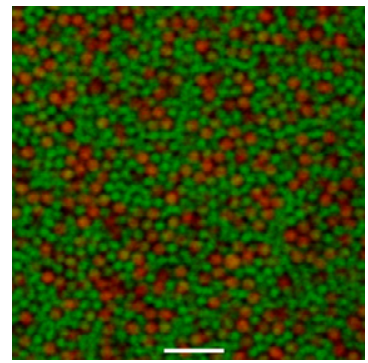

(b)
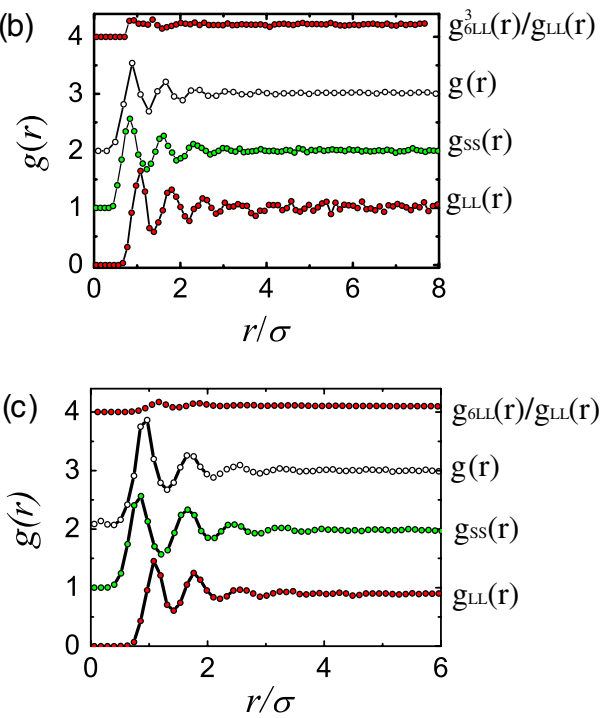

Figure 3. (a) $2 \mathrm{D}$ confocal microscope image of the $q=0.8$ system on which the 3D analysis was conducted. The absence of long-range order is clear in this image. (b) Correlation functions, $g(r)$, denote the radial distribution function without distinguishing the two species, while $g_{\mathrm{SS}}(r)$ and $g_{\mathrm{LL}}(r)$ are single-species radial distribution functions of small-small and large-large particles, respectively. (c) as (b) except that a 2D analysis was conducted. Note, however, that $g_{6 \mathrm{LL}}^{3}(r)$ is based upon the $q_{6}$ bond order parameter while $g_{6 \mathrm{LL}}(r)$ is based upon the $\phi_{6}$ order parameter.

and $N_{i}$ is the number of particles of species $i$. This is known to be directly proportional to the $3 \mathrm{D}$ volume fraction [32]. The crossover in composition reflects the images shown in figure 4 and appears to be reasonably smooth, supporting the notion of a continuous change in composition.

The structure is characterized with a (2D) radial distribution function $g(r)$, as defined above. This captures the long-ranged order of the crystal (figures 4(a) and (f), $z=6.6$ and $1.8 \mathrm{~mm}$ ), and the amorphous structures with short-ranged order at intermediate compositions, as shown in figure 6. In particular, we emphasize that the long-ranged oscillatory nature of the $g(r)$ plotted for $z=6.6 \mathrm{~mm}$ and also for $z=1.8 \mathrm{~mm}$ provide clear evidence of long-ranged order, i.e. crystallinity. By comparison, we note that, for $z=3.3 \mathrm{~mm}$, the oscillations are not apparent for $r>4 \sigma$. According to our discussion above, we conclude that this analysis has indeed demonstrated a transition from a state with long-ranged order $(z=6.6 \mathrm{~mm})$ to a state without long-ranged order $(z=3.3 \mathrm{~mm})$, followed by a second transition to a state with long-ranged order $(z=1.8 \mathrm{~mm})$. 

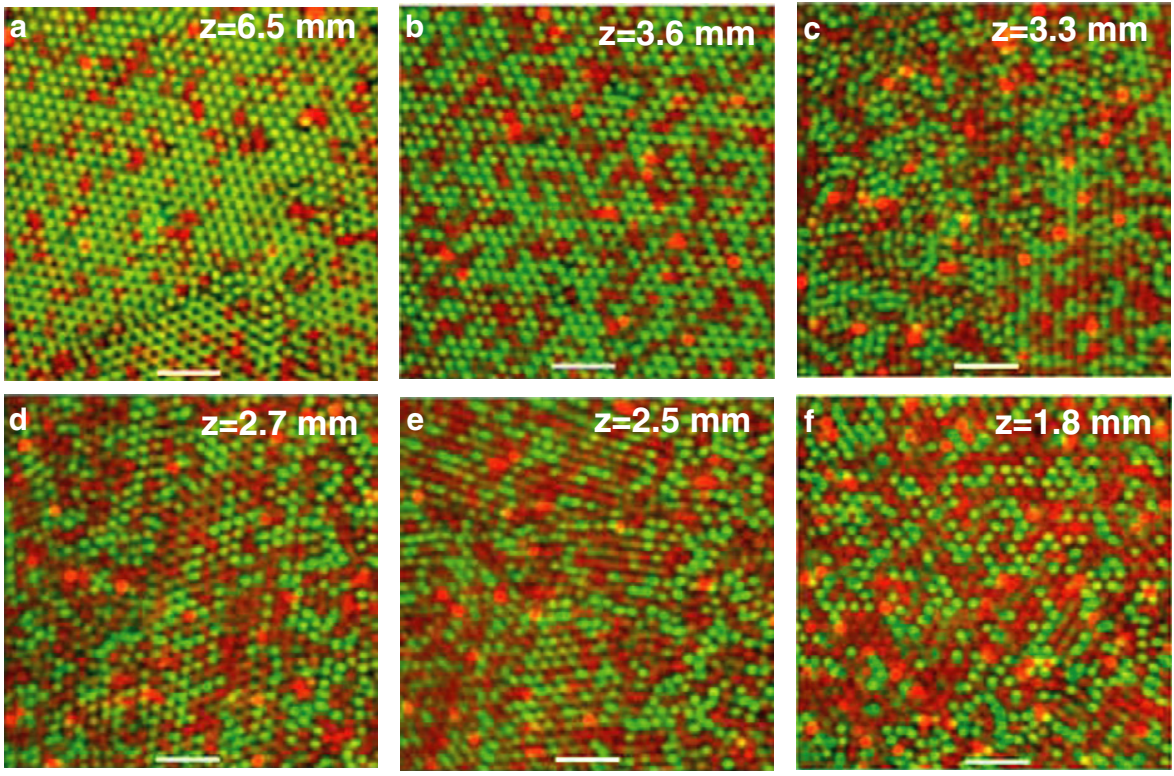

Figure 4. Confocal microscopy images in the experimental geometry shown. When the composition is dominated by one species $(z=6.5,1.8 \mathrm{~mm})$, the structure appears more ordered. More equimolar compositions suggest a reduction in long-ranged order. Larger (rhodamine-labelled) and smaller (NBD-labelled) particles are shown in red (dark) and green (bright), respectively. Bars $=10 \mu \mathrm{m}$. Here $q=0.92$.

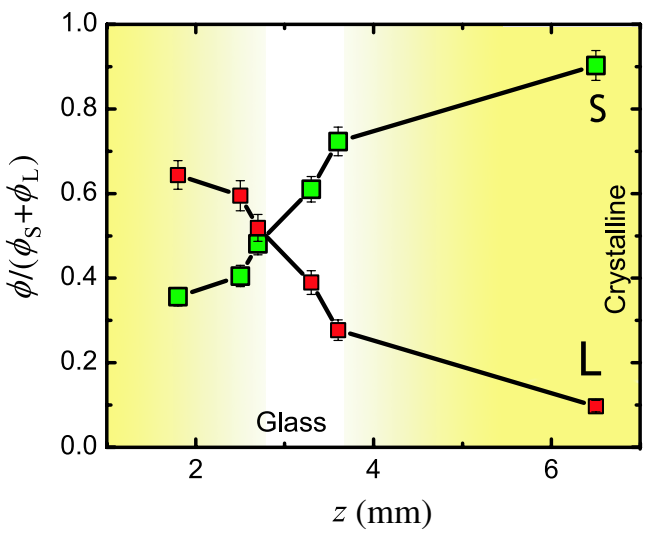

Figure 5. Relative compositional change with height, determined from coordinate tracking. Green (S) denotes small (NBD-labelled) particles and red (L) large (rhodamine-labelled). $\phi$ denotes area fraction. Shaded areas indicating crystalline regions are a guide to the eye. Here $q=0.92$.

We also consider single-species radial distribution functions $g_{\mathrm{LL}}(r)$ and $g_{\mathrm{SS}}(r)$ where the subscripts denote largelarge (rhodamine-rhodamine) and small-small (NBD-NBD) particle correlations, respectively, plotted in figure 7 . The radial distribution function results are entirely consistent with our picture of a substitutional crystal, when one species is in the majority. On the other hand, approaching equimolar mixing, we find a disordered amorphous state. Finally, when the other species is in the majority, we again find a crystalline phase.

One powerful use of real space analysis, as a tool to tackle the glass transition, lies in the manipulation of particle level information to reveal local details which are hard to access directly with other experimental techniques. Measures such as bond-orientational order parameters are especially

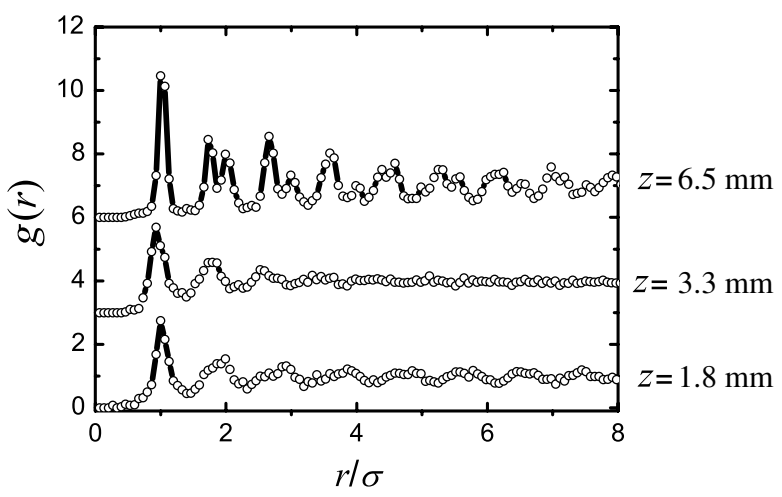

Figure 6. (2D) Radial distribution functions $g(r)$ at different heights. Here no distinction is made between large and small particles. The order decreases and then increases again upon further compositional change. $\sigma=0.5\left(\sigma_{\mathrm{S}}+\sigma_{\mathrm{L}}\right)$ is the mean colloid diameter where $\sigma_{\mathrm{S}}$ and $\sigma_{\mathrm{L}}$ are the diameters of the small and large particles, respectively. Lines are offset for clarity. Here $q=0.92$.

useful in characterizing the local structure and symmetry. In figure 8 we consider $g_{6}(r) / g(r)$. As a sensitive measure of the local crystalline environment, $g_{6}(r) / g(r)$ reveals significant crystallinity only in the region rich in small (NBD-labelled) colloids at $z=6.5 \mathrm{~mm}$, in which $\phi_{\mathrm{S}} /\left(\phi_{\mathrm{S}}+\phi_{\mathrm{L}}\right) \approx 0.9$ (figure 5).

\section{Conclusions}

We have demonstrated a method which allows us to continuously scan compositional changes in a model system of colloidal hard spheres. Both species crystallize in isolation. Upon adding a small quantity of the other species, a substitutional crystal is formed (for size ratio $q=0.92$ ). 


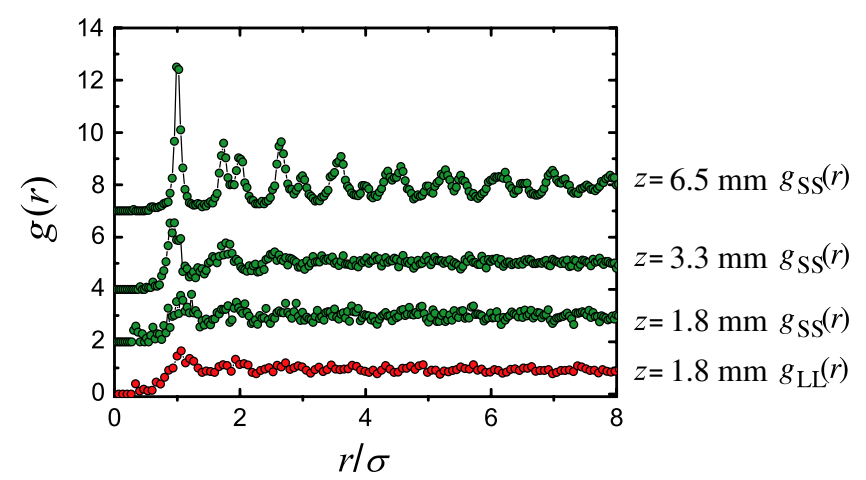

Figure 7. (2D) $g(r)$ at different heights for single species. $g_{\mathrm{SS}}(r)$ and $g_{\mathrm{LL}}(r)$ denote correlations between NBD-and rhodamine-labelled particles, respectively. Lines are offset for clarity. Here $q=0.92$.

Higher concentrations lead to the formation of a more glassy amorphous state. We have used single-particle-level real space analysis to reveal the crossover from an ordered, crystalline state to a more disordered state, which we have demonstrated with 2D $g(r)$ and $g_{6}(r)$ measurements. Thus, our method can be used for an efficient experimental determination of glassforming compositions of binary colloidal mixtures.

Furthermore, we have considered the limitations of our 2D approach. These findings have consequences for other work using confocal microscopy to study amorphous systems. For the present case, our 2D analysis is sufficient to distinguish between those states that have long-ranged order and those which do not. However, for quantitative measurements, such as oscillations in the radial distribution function close to contact, a $3 \mathrm{D}$ analysis is needed, particularly in the case of an amorphous structure. Furthermore, even in the case of crystals, not all contributions to $g(r)$ are measured in a 2D analysis, and therefore some higher-order peaks will not be present. Thus we conclude that $2 \mathrm{D}$ coordinate analysis of a $3 \mathrm{D}$ system is a qualitative, rather than quantitative measure.

We now discuss some topics for further study. A most natural extension of this work would be a full 3D analysis. In particular, this would enable a robust determination of the colloid volume fraction. For our 2D analysis, we have implicitly assumed here that the colloid volume fraction approaches close packing which, in this polydisperse system, might even exceed the random close packing value of 0.64 .

We have considered binary colloidal systems in this case. Unlike hard-sphere-like suspensions of one colloidal species, which are characterized only by the volume fraction, binary hard spheres have three parameters: the volume fractions of each species $\phi_{\mathrm{S}}$ and $\phi_{\mathrm{L}}$, along with the size ratio $q$. In this case another key parameter is the rate of sedimentation, characterized by the Peclet number $P e=\tau_{B} / \tau_{\mathrm{S}}$ where $\tau_{B}$ is the time to diffuse one radius and $\tau_{\mathrm{S}}$ is the time to sediment one radius. $\tau_{B}=3 \pi \eta \sigma^{3} / 4 k_{\mathrm{B}} T$ where $\eta$ is the viscosity and $\tau_{\mathrm{S}}=\sigma / 2 u_{0}$. Many of these parameters can be tuned and we expect crystallization in the case of suspensions of one species of reasonably monodisperse (polydispersity $\leqslant 8 \%$ ) particles for $P e \leqslant 1$. Higher sedimentation rates (higher $P e$ ) lead to disordered sediments [33]. The Peclet number may

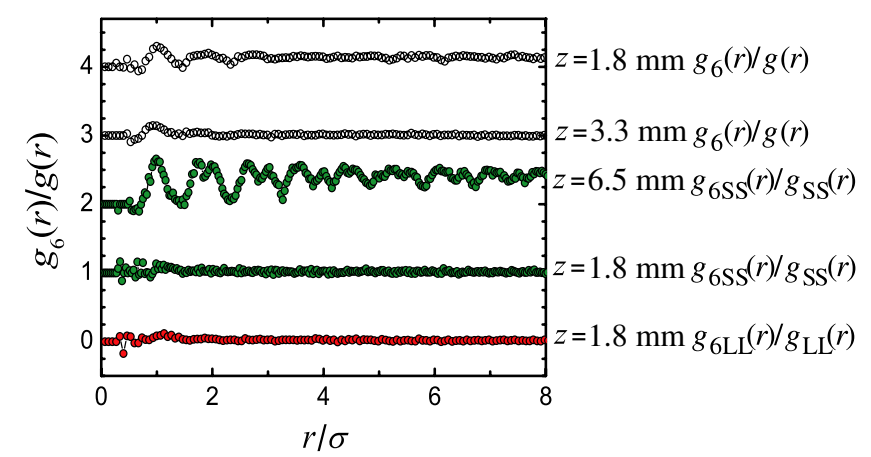

Figure 8. $g_{6}(r) / g(r)$ at different heights. $g_{6}(r)$ is sensitive to sixfold symmetry (see equation (3)). Unfilled circles do not distinguish different species, filled circles are denoted by colour. $g_{6}(r) / g(r)$ shows the decrease and increase in long-ranged order. $g_{6}(r) / g(r)$, $g_{6 \mathrm{SS}}(r) / g_{\mathrm{SS}}(r)$ and $g_{6 \mathrm{LL}}(r) / g_{\mathrm{LL}}(r)$ correspond to correlations between all particles, NBD-labelled particles and rhodamine-labelled particles, respectively. Lines are offset for clarity. Here $q=0.92$.

also be expressed in terms of the gravitational height $l_{\mathrm{G}}$ as $P e=\sigma / 2 l_{\mathrm{G}}$.

For systems in the regime we considered here, we expect the composition, at least in the disordered phase, to approach random close packing 0.64. However, two of us have previously demonstrated the use of a centrifuged colloidal dispersion to rapidly scan a continuous range of volume fractions [34]. Thus we can easily extend the approach presented here, using samples with different initial compositions, to study the full state diagram, in particular at densities lower than random close packed. Other possibilities include density matching such that the gravitational height $l_{\mathrm{G}}$ is comparable to the system size [35]. Combined with centrifugation perpendicular to gravity, this would enable a continuous range of compositions and overall concentrations to be accessed in a single sample.

Other promising approaches for this technique could be to investigate the formation of the Laves phase recently found to be the stable phase in binary hard spheres with a size ratio of around 0.8 [36].

\section{Acknowledgments}

Stephen Williams and Willem Kegel are thanked for helpful discussions. We would like to thank Didi Derks and Mirjam Leunissen for particle synthesis. This work was partly supported by a Grant-in-Aid for JSPS Fellowships for Overseas Researchers and also a Grant-in-Aid from the Ministry of Education, Culture, Sports, Science, and Technology, Japan. CPR acknowledges The Royal Society for financial support. ECMV is supported by NanoNed, a nanotechnology programme of the Dutch Ministry of Economic Affairs.

\section{References}

[1] Sciortino F and Tartaglia P 2005 Glassy colloidal systems Adv. Phys. 54 471-524

[2] Debenedetti P G 1996 Metastable Liquids Concepts and Principles (Princeton, NJ: Princeton University Press) 
[3] Widmer-Cooper A and Harrowell P 2005 On the relationship between structure and dynamics in a supercooled liquid J. Phys.: Condens. Matter 17 S4025-34

[4] Widmer-Cooper A and Harrowell P 2006 Free volume cannot explain the spatial heterogeneity of Debye-Waller factors in a glass-forming binary alloy $J$. Non-Cryst. Solids 352 5098-102

[5] Ediger M D 2000 Spatially heterogeneous dynamics in supercooled liquids Annu. Rev. Phys. Chem. 51 99-128

[6] Tanaka H 1999 Two-order-parameter description of liquids. 1. A general model of glass transition covering its strong to fragile limit J. Chem. Phys. 111 3163-74

[7] Shintani H and Tanaka H 2006 Frustration on the way to crystallization in glass Nat. Phys. 2 200-6

[8] Kawasaki T, Araki T and Tanaka H 2007 Correlation between dynamic heterogeneity and medium range order in two-dimensional glass-forming liquids Phys. Rev. Lett. 99215701

[9] Watanabe K and Tanaka H 2008 Direct observation of medium-range crystalline order in granular liquids near the glass transition Phys. Rev. Lett. 100158002

[10] Pusey P N and van Megen W 1986 Phase behaviour of concentrated suspensions of nearly hard colloidal spheres Nature 320 340-2

[11] van Blaaderen A and Wilzius P 1995 Real-space structure of colloidal hard-sphere glasses Science $\mathbf{2 7 0} 1177$

[12] Gasser U, Schofield A and Weitz D A 2003 Local order in a supercooled colloidal fluid observed by confocal microscopy J. Phys.: Condens. Matter 15 S375-80

[13] Frank F C 1952 Supercooling of liquids Proc. R. Soc. A 215 43-6

[14] Kegel W K and van Blaaderen A 2000 Direct observation of dynamic heterogeneities in colloidal hard-sphere suspensions Science 287 290-3

[15] Weeks E R, Crocker J C, Levitt A C, Schofield A and Weitz D A 2001 Three-dimensional direct imaging of structural relaxation near the colloidal glass transition Science 287 627-31

[16] Moriguchi I, Kawasaki K and Kawakatsu T 1995 Long-lived glassy states in dense nearly hard-sphere colloids J. Physique II 5 143-57

[17] Rintoul M D and Torquato S 1996 Computer simulations of dense hard-sphere systems J. Chem. Phys. 105 9258-65

[18] Martin S M, Bryant G and van Megen W 2007 Crystallization kinetics of polydisperse colloidal hard spheres: experimental evidence for local fractionation Phys. Rev. E 67061405

[19] Kob W and Andersen H C 1995 Testing mode-coupling theory for a supercooled binary Lennard-Jones mixture: the Van Hove correlation function Phys. Rev. E 51 4626-41

[20] Williams S R and van Megen W 2001 Motions in binary mixtures of hard colloidal spheres: melting of the glass Phys. Rev. E 64041502
[21] Martin S, Bryant G and van Megen W 2005 Crystallization kinetics of polydisperse colloidal hard spheres. II. binary mixtures Phys. Rev. E 71021404

[22] Hamanaka T and Onuki A 2007 Heterogeneous dynamics in polycrystal and glass in a binary mixture with changing size dispersity and composition Phys. Rev. E 75041503

[23] van Blaaderen A 1997 Quantitative real-space analysis of colloidal structures and dynamics with confocal scanning light microscopy Prog. Colloid Polym. Sci. 104 59-65

[24] Biben T and Hansen J-P 1993 Localized density profiles in binary colloidal suspensions Mol. Phys. 80 853-9

[25] Yethiraj A and van Blaaderen A 2003 A colloidal model system with an interaction tunable from hard sphere to soft and dipolar Nature 421 513-7

[26] Royall C P, van Roij R and van Blaaderen A 2005 Extended sedimentation profiles in charged colloids: the gravitational length, entropy, and electrostatics J. Phys.: Condens. Matter 17 2315-26

[27] Bosma G, Pathmamanoharan C, de Hoog E H A, Kegel W K, van Blaaderen A and Lekkerkerker H N W 2002 Preparation of monodisperse, fluorescent PMMA-latex colloids by dispersion polymerization J. Colloid Interface Sci. 245 292-300

[28] Crocker J C and Grier D G 1995 Methods of digital video microscopy for colloidal studies J. Colloid Interface Sci. 179 298-310

[29] Royall C P, Dzubiella J, Schmidt M and van Blaaderen A 2007 Nonequilibrium sedimentation of colloids on the particle scale Phys. Rev. Lett. 98188304

[30] Binder K, Sengupta S and Nielaba P 2002 The liquid-solid transition of hard discs: first-order transition or Kosterlitz-Thouless-Halperin-Nelson-Young scenario? J. Phys.: Condens. Matter 14 2323-33

[31] Steinhardt P J, Nelson D R and Ronchetti M 1983 Bond-orientational order in liquids and gases Phys. Rev. B 28 784-805

[32] Leocmach M, Royall C P and Tanaka H 2008 Phase separation and sedimentation in binary colloid+polymer mixtures: crystal zeppelins, in preparation

[33] Russel W B, Saville D A and Schowalter W R 1989 Colloidal Dispersions (Cambridge: Cambridge University Press)

[34] Royall C P, Leunissen M E and van Blaaderen A 2003 A new colloidal model system to study long-range interactions quantitatively in real space J. Phys.: Condens. Matter 15 S3581-96

[35] Royall C P, Louis A A and Tanaka H 2007 Measuring colloidal interactions with confocal microscopy J. Chem. Phys. 127044507

[36] Hynninen A-P, Thijssen J H J, Vermolen E C M, Dijkstra M and van Blaaderen A 2007 Self-assembly route for photonic crystals with a bandgap in the visible region Nat. Mater. 6 202-5 